\title{
The Optimization Design of an Ionic Liquid Composite Material for Nitrogen Removal
}

\author{
Bao-you Liua and Tao Wang \\ Key Laboratory of pollution prevention and control in Hebei Province. College of Environmental Science and Engineering, Hebei University of Science and Technology, Shijiazhuang Hebei 050018, China
}

\begin{abstract}
A kind of EMIMTA ionic liquid was prepared. The viscosity and the absorption amount of NO2 of EMIMTA-TEA composites were measured in different proportion and different temperature. Origin software was used to fit the mathematical equation of the relationship between the viscosity, the absorption amount of NO2 and the mole fraction of the ionic liquid. By the lingo software, the better proportion of Ionic liquid and triethanolamine is optimized. The results show that both the viscosity and the absorption amount of NO2 decrease with the increase of the mole fraction of EMIMTA, which are in accordance with the two order polynomial equation. In order to obtain better absorption amount of NO2 and lower viscosity of composites, taking the absorption amount of $\mathrm{NO} 2$ / viscosity maximization as the optimization goal, the viscosity and absorption amount equation NO2 as constraint conditions, the mathematical model of nonlinear programming is established and solved. The results show that when the molar ratio of the ionic liquid is 0.4091 , the viscosity of the composite is $47.5 \mathrm{mPa} / \mathrm{s}$, and the absorption amount of NO2 is $0.7744 \mathrm{~g}$. g-1. This paper has some guiding significance for the optimization design of ionic liquid composites.
\end{abstract}

\section{Introduction}

Ionic liquid, as a kind of free combination salt of anion and cation, can be used as functional materials and play an important role in many fields. The ionic liquid showed different performance indexes, through physical and chemical reaction directly acting on the process of using, achieving the effect of the same with the traditional materials by its mechanical properties and chemical properties[1]. Moreover, under the same experimental conditions, the effect is better than the traditional materials, and it is an attractive alternative material. Compared to the traditional functional materials, ionic liquids have the characteristics of low vapor pressure, wide temperature range and high chemical stability, thus

a Corresponding author: liuzhang544@163.com its effect is less affected by the environment, with a wide application range. In some extreme conditions, such as high pressure, vacuum, high temperature, low temperature, etc, the ionic liquid functional material can adapt to different temperature and pressure, and the effect is not affected by the change of temperature or pressure, and can carry out the stable and adequate relevant reaction. Based on this, ionic liquids can be used under different conditions, which is one of the important reasons for used in many fields as a functional material. It has a wide range of applications, and provide a feasibility study for the new field subject. Ionic liquid is composed of anions and cations, which can lead to a variety of chemical reactions, therefore, it has a good solubility for acid gas. Compared with traditional gas absorption materials, in the process of absorbing and 
desorbing gas, the thermal stability and low vapor pressure of Ionic liquid can avoid the problem of volatile. Ionic liquid is a new type absorbent of environmental protection, which shows great potential in the field of environmental protection. Up to now, there have been some reports on the absorption of acid gases in flue gas by ionic liquids. For example, Liu[2] prepared 5 kinds of low eutectic ionic liquids of acetamide-inorganic salts that have good absorption ability to $\mathrm{SO} 2$ gas, and the absorption capacity of SO2 can reach up to $0.554 \mathrm{~g} / \mathrm{g}$ $\left(20{ }^{\circ} \mathrm{C}, 1.01 * 10^{5} \mathrm{~Pa}\right)$, the recovered ionic liquid can be reused for at least 5 times. Duan reported that caprolactam / four Butyl Ammonium chloride ionic liquid absorbed NOx, NMR results show that the adsorption and desorption of NO and NO2 is reversible in ionic liquids, providing the possibility for the removal of $\mathrm{NO} 2[3]$.

Lingo is a set of software packages for solving optimization problems, developed by Lindo systems company. Lingo is widely used in teaching, scientific research and industry because of its fast execution speed, easy input, simple solution process in analyzing mathematical programming problems[4]. Lingo is mainly used to solve linear programming, nonlinear programming, transportation planning, dynamic programming, goal programming and integer programming problem. And Lingo can also be used to solve the linear or nonlinear equations and the roots of algebraic equations. The biggest feature of the Lingo software is that it allows the variables in the optimization model is an integer, also known as integer programming. Actually, Lingo is a modeling language to solve optimization problems. In order to facilitate the user to establish the optimization model, Lingo also provides a number of commonly used functions for users to call. In addition, it also provides the interface with other data files, which makes it easier to input, solve and analyze large-scale optimization problems. On the basis of the existing research on the ionic liquid for desulfurization and denitration, in view of the advantages and disadvantages of ionic liquids denitrification in flue gas, preparation of ionic liquid composite absorbing material through a mixture of ionic liquid and organic alkali absorber-triethanolamine, the relationship between the viscosity of ionic liquid and organic alkali in different proportions and the absorption amount of NO2 were measured respectively, and the equation of the relationship between them was established by Origin in this paper. Considering that Lingo software has been successfully applied to the optimization design of composite materials[5], taking absorption amount / viscosity as the optimization objective, the optimum proportion of composite material was obtained by Lingo software to achieve the purpose of larger absorption capacity, lower viscosity and less energy consumption. This study can provide some theoretical guidance for the industrial application of ionic liquids, so as to realize NO2 treatment scheme of the energy saving and environmental protection.

\section{Experimental}

\subsection{Instruments and reagents}

The main instruments are $\mathrm{Fu}$ Liye transform infrared spectroscopy, Tencer 27, German Bruker company; Nuclear magnetic resonance spectromete, AVANCE II 400M, German Bruker company.The reagents used in this study were analysis pure ( $\geq 99 \%$ ).

\subsection{Experimental procedure}

\subsubsection{Preparation of ionic liquid EMIMTA}

30mL 1-methylimidazolium and $90 \mathrm{~mL}$ bromoethane are mixed in $250 \mathrm{~mL}$ round bottom flask, heating for $8 \mathrm{~h}$, getting oily liquid, then cooling to obtain white solid. Acetonitrile is used for dissolving and filtering; filtrate is added with ethyl acetate to obtain white solid, dissolved in acetonitrile again,and then filtering. Recrystallization products were dried under vacuum for 36 hours,after that, 1- ethyl -3- methyl bromide (EMIMBr) was obtained. Take 19.1g EMIMBr and 22.4g three fluorine acetic acid silver, added to $200 \mathrm{~mL}$ acetonitrile, after stirring, filtering to remove the silver bromide precipitation, rotary evaporation to remove acetonitrile. When the product was dried under vacuum at $60^{\circ} \mathrm{C}$, 1- ethyl -3methyl imidazole three acetic acid salt was obtained. The structure of EMIMTA ionic liquid was characterized by Fourier transform infrared (FT-IR) spectroscopy and nuclear magnetic resonance spectroscopy (1H NMR). 
The results shown that the purity was more than $99 \%$.

\subsubsection{The viscosity of EMIMTA-TEA}

In the calculation of the viscosity, the density value used can be measured by using GB4472-84 method-Specific gravity bottle method $(10 \mathrm{~mL})$. The density was measured by the precision electronic balance (Precision is $\pm 10^{-4} \mathrm{~g}$ ), using the pure water bottle calibration before determination. Super thermostatic bath with accuracy of $\pm 10^{-2} \mathrm{~K}$ was used in temperature controlling. The viscosity was measured by Pinshi viscometer. Calibration liquid were glycerol, ethylene glycol and water respectively. Each experiment was repeated three times, each time interval of $10 \mathrm{~min}$, calculating viscosity by arithmetic mean.

\subsubsection{EMIMTA-TEA absorption of NO2}

Absorption device and process reference Literature 2 . Temperature controlling accuracy is $\pm 10^{-1} \mathrm{~K}$, weighing accuracy is $\pm 10^{-2} \mathrm{~g}$. In the process of absorption, the quality of $\mathrm{NO} 2$ gas is measured by the electronic balance every $10 \mathrm{~min}$, until the mass change range is less than $1 \%$. Each experiment was repeated three times and the arithmetic mean was taken as the amount of absorption.

\subsubsection{Optimization process}

Taking the ratio of absorption and viscosity as objective function, taking the fitted equation of viscosity and X1 (molar fraction of ionic liquid) and the fitted equation between absorption amount and X1 (mole fraction of ionic liquid) as the main constraint condition, the mathematical model of nonlinear programming is established and solved by lingo11.0.

\section{Results and Discussion}

\subsection{Viscosity of EMIMTA-TEA Composites}

Table 1 Viscosity ( $\mathrm{mPa} \cdot \mathrm{s}$ ) of EMIMTA-TEA solution with different EMIMTA molar ratios $\left(\mathrm{X}_{1}\right)$ at different temperatures $(\mathrm{T}, \mathrm{K})$

\begin{tabular}{|c|c|c|c|c|c|}
\hline $\begin{array}{c}\mathrm{X}_{1} \\
\mathrm{~T}\end{array}$ & 303.15 & 313.15 & 323.15 & 333.15 & 343.15 \\
\hline 0 & 405.92 & 207.24 & 108.69 & 60.25 & 36.29 \\
\hline 0.1128 & 320.4 & 149.99 & 86.1 & 52.38 & 32.87 \\
\hline 0.2006 & 234.79 & 117.78 & 65.22 & 40.93 & 27.13 \\
\hline 0.3337 & 120.61 & 69.56 & 39.08 & 25.55 & 18.02 \\
\hline 0.4299 & 67.35 & 41.65 & 26.19 & 17.46 & 12.69 \\
\hline 0.5058 & 44.99 & 28.61 & 19.73 & 13.72 & 10.47 \\
\hline 0.6051 & 37.59 & 23.83 & 17.74 & 12.32 & 9.27 \\
\hline 0.6965 & 37.59 & 25.49 & 17.6 & 12.6 & 9.64 \\
\hline 0.7994 & 35.58 & 26.28 & 17.44 & 12.36 & 9.74 \\
\hline 0.9001 & 30.63 & 19.88 & 15.61 & 11.64 & 9.41 \\
\hline 1.0000 & 26.56 & 17.24 & 13.04 & 10.39 & 9.34 \\
\hline
\end{tabular}

The viscosity of EMIMTA-TEA mixture was determined at different temperatures (303.15 K-343.15 K). The viscosity of EMIMTA-TEA solution at different temperature and molar ratio is shown in Table 1. The viscosity of EMIMTA-TEA solution decreased with the increase of EMIMTA molar ratio,and the lower the molar ratio, the more significant the degree of decline. With $303.15 \mathrm{~K}$ as an example, when the molar ratio of $\mathrm{X} 1$ is less than 0.5 , with the increase of the molar ratio, the viscosity of EMIMTA-TEA solution decreased rapidly from $405.92 \mathrm{mPa} \cdot \mathrm{s}$ to $44.99 \mathrm{mPa} \cdot \mathrm{s}$, when the molar ratio is more than 0.5, the viscosity of EMIMTA-TEA decreased slowly from $37.59 \mathrm{mPa} \cdot \mathrm{s}$ to $26.56 \mathrm{mPa} \cdot \mathrm{s}$. In addition, when the temperature is higher, such as 333.15 $\mathrm{K}$ and $343.15 \mathrm{~K}$, the viscosity of EMIMTA-TEA solution decreases more gentle than $303.15 \mathrm{~K}$. The results shown that the viscosity of EMIMTA-TEA solution with different EMIMTA molar ratios was lower at high temperature. Thus, the viscosity can be controlled by adjusting the temperature.

\subsection{NO2 absorption amount of EMIMTA-TEA Composites}

Under atmospheric pressure, at the same temperature point and different distribution ratio groups, ionic liquid-Triethanolamine solution (EMIMTA-TEA) has different absorption amount for NO2. When the temperature is the same, with the increase of the content of triethanolamine in the EMIMTA-TEA solution system, and the absorption amount of NO2 was gradually 
increased, which was higher than that of pure EMIMTA.

Table 2 NO2 absorption of EMIMTA-TEA solution $\left(\mathrm{g} \cdot \mathrm{g}^{-1}\right)$ with different EMIMTA molar ratios $\left(\mathrm{X}_{1}\right)$ at different temperatures( $\mathrm{T}, \mathrm{K})$

\begin{tabular}{|c|c|c|c|c|c|}
\hline $\begin{array}{c}\mathrm{T} \\
\mathrm{X}_{1}\end{array}$ & 0.0000 & 0.4299 & 0.5006 & 0.6636 & 1.0000 \\
\hline 303.15 & 1.0562 & 0.8570 & 0.8119 & 0.7687 & 0.6795 \\
\hline 313.15 & 0.8846 & 0.7739 & 0.7409 & 0.7062 & 0.6233 \\
\hline 323.15 & 0.7645 & 0.6757 & 0.6518 & 0.6261 & 0.5525 \\
\hline 333.15 & 0.6506 & 0.5820 & 0.5683 & 0.5440 & 0.4933 \\
\hline 343.15 & 0.5227 & 0.4753 & 0.4725 & 0.4594 & 0.4394 \\
\hline
\end{tabular}

\subsection{Model optimization better ratio}

Under normal pressure, when temperature is the same, the absorption amount of $\mathrm{NO} 2$ by ionic liquids-triethanolamine solution (EMIMTA-TEA) of different groups was different. At the same temperature, with the increase of the triethanolamine content in the EMIMTA-TEA solution, the absorption amount of NO2 was gradually increased, which was higher than that of pure EMIMTA.

Table 3 NO2 absorption amount $\left(\mathrm{g} \cdot \mathrm{g}^{-1}\right)$ byEMIMTA-TEA

Solution with different EMIMTA molar ratio $\left(\mathrm{X}_{1}\right)$ and temperature( $\mathrm{T}, \mathrm{K})$

\begin{tabular}{|c|c|c|c|c|c|}
\hline $\begin{array}{c}\mathrm{T} \\
\mathrm{X}_{1}\end{array}$ & 0.0000 & 0.4299 & 0.5006 & 0.6636 & 1.0000 \\
\hline 303.15 & 1.0562 & 0.8570 & 0.8119 & 0.7687 & 0.6795 \\
\hline 313.15 & 0.8846 & 0.7739 & 0.7409 & 0.7062 & 0.6233 \\
\hline 323.15 & 0.7645 & 0.6757 & 0.6518 & 0.6261 & 0.5525 \\
\hline 333.15 & 0.6506 & 0.5820 & 0.5683 & 0.5440 & 0.4933 \\
\hline 343.15 & 0.5227 & 0.4753 & 0.4725 & 0.4594 & 0.4394 \\
\hline
\end{tabular}

\subsection{Model optimization}

Taking $313.15 \mathrm{~K}$ as an example, the absorption amount of $\mathrm{NO} 2$ increased with the increase of TEA molar ratio in EMIMTA-TEA solution. It is concluded that increasing TEA can increase the NO2 absorption amount of ionic liquids. However, the pure triethanolamine viscosity is larger, when the temperature is $313.15 \mathrm{~K}$, the viscosity is $207.24 \mathrm{mPa} / \mathrm{s}$, about 12 times of pure ionic liquid of the same conditions. With the increase of molar ratio of triethanolamine solution in EMIMTA-TEA, the viscosity of the solution gradually increases. When we are in the pursuit of higher absorption amount of EMIMTA-TEA solution, it is necessary to avoid the impact of greater viscosity. Therefore, through fitting the relationship between the molar ratio of EMIMTA and the absorption and viscosity of EMIMTA-TEA solution, this section establishes a mathematical model, using Origin and LINGO software to optimize. Then we can obtain EMIMTA-TEA molar ratio, under high absorption and low viscosity conditions. It can provide theoretical basis for practical production and application.

At $313.15 \mathrm{~K}$, the viscosity and absorption of EMIMTA-TEA were fitted by polynomial equation. According to the fitting equation, the mathematical model is established and optimized. The results shown, when the molar ratio of EMIMTA in the EMIMTA-TEA solution was 0.4091 , the absorption was better, and the corresponding viscosity was $47.50 \mathrm{mPa} / \mathrm{s}$, and the absorption was about $\mathrm{g}$.g-1. It also shows that the EMIMTA-TEA solution in this ratio can guarantee a higher absorption, but also reduce the viscosity. When EMIMTA-TEA is used to absorb NO2 in the industrial production, the cost is low and the operation is simple.

\section{Conclusions}

1- ethyl -3- methyl imidazolium based ionic liquid EMIMTA was prepared, the structure was characterized by Fourier transform infrared spectroscopy (FT-IR) and nuclear magnetic resonance spectroscopy $\left({ }^{1} \mathrm{H}\right.$ NMR). The viscosity of EMIMTA-TEA ionic liquid composite was measured at different temperatures (303.15 K-343.15 K). At the same temperature, the viscosity of EMIMTA-TEA solution decreased with the increase of EMIMTA molar ratio. At same EMIMTA molar ratio, the viscosity of EMIMTA-TEA solution decreases with the increase of temperature. Under atmospheric pressure, when the temperature range is $303.15 \mathrm{~K}-343.15 \mathrm{~K}$, NO2 gas absorption of EMIMTA-TEA ionic liquid composites is measured. At the same temperature, NO2 absorption amount gradually increased with the increase of EMIMTA-TEA (TEA) ratio of triethanolamine solution. The mathematical model is established and optimized by 
software calculation. The results showed that when the molar ratio of EMIMTA was 0.4091 , the viscosity was $47.50 \mathrm{mPa} / \mathrm{s}$, and the absorption was about g.g-1. At this time, the EMIMTA-TEA composite is the best absorbent with good absorption and low viscosity.

\section{References}

[1] C. Chiappe, C. S. Pomelli. Point-Functionalization of Ionic Liquids: An Overview of Synthesis and Applications. EUR J ORG CHEM, (2014), 2014(28): 6120-6139

[2] B. Y. Liu, F. X. Wei, J. J. Zhao, et al. Characterization of Amide-Thiocyanates Eutectic Ionic Liquids and Their Application in SO2 Absorption. RSC advance,(2013), 3(7): 2470-2476

[3] E.H. Duan, B. Guo, D. D. Zhang, et al. Absorption of $\mathrm{NO}$ and NO2 in Caprolactam Tetrabutyl Ammonium Halide Ionic Liquids. J Air Waste Manage Assoc, (2011), 61(12): 1393-1397

[4] J. X. Xie, Y..Xue, optimization modeling and LINDO $L I N G O$ software [M], Beijing: Tsinghua University press, (2005.7)

[5] H. P. Han, Y. He, H. C. Lin, et al., the application of uniform design in rubber nano composite material preparation technology, Anhui Agricultural Sciences,(2008), 363064-3066.

\section{Acknowledgments}

The authors gratefully acknowledge Natural Science Foundation of Hebei Province (2015208122) and environment engineering key subject construction of Hebei Province for their financial supports. 